\title{
A Kohonen Network Model for Performance Evaluation of Asphalt Concrete Pavement
}

\author{
Qin Zhi-bin*, Zhang Wen-ting, Zheng Hui and Wang Kang
}

School of Changsha University of Science and Technology, Changsha, Hunan, 410114, P.R.China

\begin{abstract}
Analyses the current road pavement maintenance technical specification, put forward a new evaluation method for maintenance and overlay of old asphalt pavement. Considering the uncertainty and complexity in the process of performance evaluation, and based on the entropy and the Kohonen network theory, the performance evaluation system is established in Kohonen network of fuzzy entropy method, combine the expert's weight and entropy method to confirm the weight, in order to reflect the subjectivity of classification and objectivity of measured data at the same time.
\end{abstract}

Keyword: Road engineering; Asphalt pavement performance; Kohonen network; Fuzzy entropy.

In the current "Chinese technical specifications for maintenance of highway asphalt pavement" [1], the sub-item state of road's performance evaluation content including the road surface breakage condition, the riding quality, the intensity and the anti-slippery performance, the indicator which uses corresponds separately for pavement condition index (PCI), road quality index (RQI), surface strength index (SSI), sideway force coefficient (SFC or BPN), composite evaluation index (PQI) to be used the pavement comprehensive assessment. Based on the above various subitems indicator evaluation results to count, and corrected weighting for each evaluation rank's intermediate quantity [2-5]. This evaluation method has 3 problems [6-9]: the differences of the road condition within the same rank is neglected easily or cannot obtain manifests. When the difference of the sub-item indicator is not big, sometimes the sub-item rank's determination can enable the $P_{P Q I}$ value to change greatly. The determined of pavement overall evaluation rank is not enough direct. Therefore, it was difficult to compare among different road sections, also difficult to determine and optimization.

The pavement performance is a multifactor comprehensive evaluation problem, and will inevitably contain a synthetic judgment of various factors in the process of all kinds of judgment [10-12], so, there will have certain human difference, and the fuzzy phenomenon both between "superior" and "good" and "good" and "medium". Therefore, this paper based on the fuzzy entropy and the Kohonen network method, establish an evaluation model of asphalt pavement performance which used various indicator performance parameters, provide a new way for evaluating and forecasting the environmental impact assessment of asphalt pavement [13-15].

\section{SAMPLE DISTINCTION}

According to "Chinese technical specifications for maintenance of highway asphalt pavement" [1], scope of sample value was determined as shown in Table 1. When various indicators achieve the marginal value, it will have a serious influence to the road safety operation, therefore, when there was an indicator's actual value surpass the marginal value in the indicator system, this road section will be determined directly as the bad one, and the influence of other indicators will no longer be considered.

\section{WEIGHTS DETERMINATION}

When the environmental impact assessment was based on the Kohonen network theory, it was extremely important to define the indicator weight which usually influenced the result's objectivity. In view of subjective factors of experts' knowledge, experience and value judgment, and objective information characters of actual measuring data, it defined the index weight by combining subjective weight method (analytic hierarchy processanalytical hierarchy AHP) with objective weight method (entropy method), so as to objectively and completely reflect both the important of evaluation index and actual condition of problem.

\subsection{Subjective weight}

According to the asphalt pavement maintenance and technical specification recommended value, the subjective

Table 1. Target value and marginal value of evaluation index.

\begin{tabular}{|c|c|c|}
\hline Evaluation index & Target value & Marginal value \\
\hline \hline Pavement condition index PCI & 100 & 30 \\
\hline Surface strength index SSI & 1.4 & 0.4 \\
\hline Road quality index RQI & 10 & 2 \\
\hline Sideway force coefficient SFC & 0.7 & 0 \\
\hline
\end{tabular}

2015 Bentham Open 
weight is $w_{i}^{\prime}$.

$$
w_{i}=\left(w_{1}, w_{2}, w_{3}, w_{4}\right)
$$

There are lots of method to determine index weight, such as expert investigation method, analytic hierarchy process (AHP), etc, and the AHP is chosen. The acquired weight set is showed:

$$
w=\left(w_{1}, w_{2}, w_{3}, w_{4}, w_{5}\right)
$$

\subsection{The objective weight is determined by entropy method}

In the information theory, the entropy value which reflected the disordering degree of the information may use to measure the size of the information, one index carried the more information, indicating that the more useful this index have in making the decision, while the entropy value is smaller, namely the system's disordering degree is smaller. Therefore the order degree and effectiveness can be evaluated by the information entropy, namely the judgment matrix made up by the evaluating indicator value determined the weight of each evaluating indicator. Its main computation step is as follows:

Generally all the indexes are normalized to unit interval $0-1$. In order to reflecting critical effect of target state and index, the membership degree in fuzzy mathematics is introduced in standardization.

Positive indicator: bigger always better, use upper semitrapezoid distribution function for standardization.

$N\left(v_{i}\right)=\left\{\begin{array}{lc}0 & v_{i} \leq C\left(v_{i}\right) \\ \frac{v_{i}-C\left(v_{i}\right)}{T\left(v_{i}\right)-C\left(v_{i}\right)} & C\left(v_{i}\right)<v_{i}<T\left(v_{i}\right) \\ 1 & v_{i} \geq T\left(v_{i}\right)\end{array}\right.$

Negative indicator: smaller always better, lower semitrapezoid distribution function for standardization.

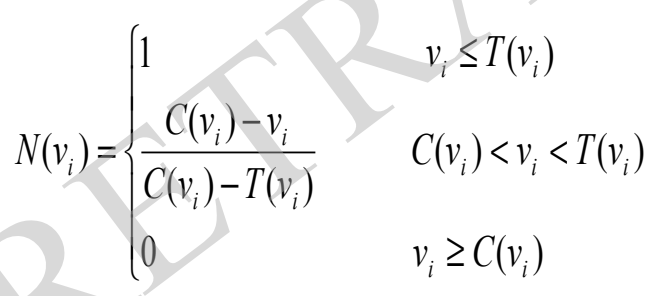

Where $v_{i}, C\left(v_{i}\right), T\left(v_{i}\right), N\left(v_{i}\right)$ are measured value, critical value, target value and standardized value of index $i$ respectively. In this paper, the target value and marginal value of evaluation index shown in Table $\mathbf{1}$.

The membership, which is based pending samples and each classification standards' lower bound for equation (1), is standardized, then obtain $r_{j i}$, and calculate follow equations (2) to (5).

$k=\frac{1}{\ln (m)}$

$$
\begin{aligned}
& e_{i}=-k \sum_{i=1}^{m} r_{j i} \ln \left(r_{j i}\right) \\
& h_{i}=1-e_{i} \\
& w_{i}=\frac{h_{i}}{\sum_{i=1}^{n} h_{i}}
\end{aligned}
$$

\subsection{Comprehensive weights}

$$
w_{i}=\frac{w_{i}^{\prime} w_{i}^{\prime \prime}}{\sum_{i=1}^{n} w_{i}^{\prime} w_{i}^{\prime \prime}}
$$

\section{KOHONEN NETWORK MODEL OF MULTIPLE FACTORS COMPREHENSIVE EVALUATION}

Kohonen network is proposed by Kohonen Professor of the Fenlands Heroin University in the 1987's. This network simulates brain characteristics of the self-organizing system function. It is a competitive learning network, which can self-organize study without supervision, so it also known as self-organizing neural network, which in recent years has been widely used in the classification model. Internet users only need to provide learning samples, rather than provide an ideal output, the network according to the input samples for self-organization, and dividing its to the corresponding model category.

\subsection{Network structure}

Kohonen network structure as shown in Fig. (1).

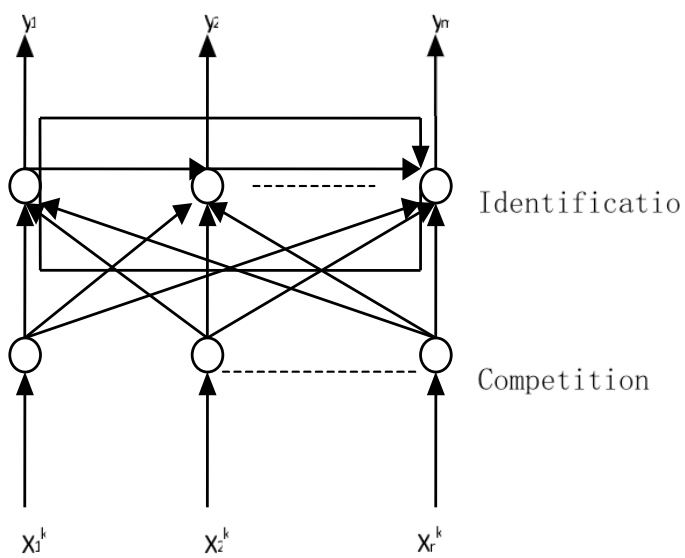

Fig. (1). Structure of Kohonen network.

The network by the competition level (competition study, choice competition "wins" neuron) and the recognition level (pattern classification) is composed, the vector $X^{k}$ input sample for the group $k$, the vector $Y$ is the output category, between the neuron joins completely mutual connection. 
Table 2. Examination sampled data and cluster table

\begin{tabular}{|c|c|c|c|c|c|c|}
\hline Evaluating indicator & Sample & Superior & Good & Median & Inferiority & Bad \\
\hline \hline Pavement condition index PCI & 69 & 92.5 & 77.5 & 62.5 & 47.5 & 35 \\
\hline Surface Strength index SSI & 7.0 & 1.2 & 0.915 & 0.745 & 0.58 & 0.45 \\
\hline Road qulity index RQI & 0.4 & 9.25 & 7.75 & 6.25 & 4.75 & 3 \\
\hline Sideway force coefficient SFC & 0.33 & 0.6 & 0.45 & 0.35 & 0.25 & 0.1 \\
\hline Cluster center category & III & I & II & III & IV & V \\
\hline
\end{tabular}

\subsection{Learning process}

After the external input mode emerges, all neurons in the network start to work at the same time, the connection right vector between them trying to imitate the input signal to achieve the goal of self-organizing network. This selforganization learning process includes two parts: First, select the best match neurons, and the second, the right vectors adaptive changes in the updating process.

Continental distance Kohonen network use the criteria as a competitive win, when the input signal and neuronal best match, the distance between them for Continental

$\left\|X-W_{c}\right\|=\min _{i}\left\{\left\|X-W_{i}\right\|\right\}$

Where $X$ for the input vector of input space $R^{n}, W_{i}$ for the corresponding weight vector.

For each input, the network only adjust part of weights, which let weight vector close to the input vector or deviate from the input vector. This adjustment process is the competitive learning. With continuous learning, all weights vector are separated from each other in the input vector space, forming their own classification mode which on behalf of their input space respectively.

\section{EXAMPLE}

Firstly, use the operating highway's measured data of one province as the sample (input membership degree value), according to the above method of computer programming to learning (set classified rank into five kinds in advance), and obtains the cluster center. Then use the measured data form literature (cf. Table 2) and the judged with the mid-value of " superior, good, median, inferiority, bad" form the present "Chinese technical specifications for maintenance of highway asphalt pavement", its result see Table 2:

The Table 2 shown that the cluster center category is respectively judged to be superior, good, median, inferiority, bad, and the sample is judged to be median, consistent with the literature conclusion.

\section{CONCLUSION}

This paper propose the comprehensive weight method which consider the subjectivity of classification and effectiveness of the measured data, more objectively and comprehensively reflect the importance of every evaluation index and the actual conditions of the problem. The application results show that this method is simple to use, evaluation results exact and reasonable, for the performance evaluation of the cement road surface provides a new method.

In this paper, using the method requires relatively rich samples. If sampling is limited, too narrow sampling samples, we can not fully reflect the actual information, the cluster center position would lack of standards. To attain the desired cluster center, the need for more sections (fully reflect the impact of urban traffic environment characteristics of the atmosphere), more time traffic monitoring of the environment, in order to obtain a large number of measured data as a learning sample, which is conducive to improve the credibility of classification.

\section{CONFLICT OF INTEREST}

The author confirms that this article content has no conflict of interest.

\section{ACKNOWLEDGEMENTS}

This project is financially supported by Key Laboratory of Highway Engineering Ministry of Education, PRC (kfj140201).

\section{REFERENCES}

[1] Technical specifications for maintenance of highway asphalt pavement, JTJ073.2-2001.

[2] J. Hong-yan, and D. Jing-liang, "Matter element model for evaluation pavement condition", Journal of Chang' an University (Natural Science Edition), vol. 25, no. 1, pp. 29-33, 2005.

[3] L. Qi-tian, Y Qin-long, and L. Fu-shun, "introduction of whole lifecycle cost analysis for road engineering", Journal of Highway and Transportation Research and Development, vol. 27, no. 9, pp. 155158, 2010.

[4] Z. Sheng, "Pavement performance evaluating model by using RBF", Journal of Highway and Transportation Research and Development, vol. 25, no. 3, pp. 23-26,54, 2008.

[5] L. Gong-yun, and H. Xiao-rming, "Comparative application of three methods for selecting pavement alternatives", Journal of Highway and Transportation Research and Development, vol. 24, no. 1, pp. 34-38, 2007.

[6] Z. Jian-nin, W. Hai-jun, and D. Hai-long, "Preventive maintenance of asphalt pavements based on effectiveness models", Journal of Highway and Transportation Research and Development, vol. 23, no. 12 , pp. $1-5,2006$.

[7] C. Peng, S. Li-jun, and H. Xiao, "Perpetual pavements on high-grade highway", Journal of Highway and Transportation Research and Development, vol. 23, no. 10, pp. 10-14, 2006.

[8] S. Yan, "Pattern recognition of mechanical fault based on Kohonen neural network", Journal of Traffic and Transportation Engineering, vol. 2, no. 2, pp. 55-59, 2002. 
[9] W. Yun-song, "Fault diagnosis of diesel based on neural network", Jorunal of Traffic and Transportation Engineering, vol. 3, no. 4, pp. 44-47, 2003.

[10] H. Xia-guang, and W. Bing-gang, "Application of two genetic algorithms based method in pavement performance synthetic evaluation", Journal of Chang' an University (Natural Science Edition), vol. 22, no. 2, pp. 6-9, 2002.

[11] F. Dong-sheng, and C. Yang-yang, "Asphalt pavement performance evaluation based on BP neural network", Transport Standardization, vol. 10, pp. 93-96, 2009.

[12] Y. Ke-zhen, W. Jian-liang, and Z. Jin-dao, "Multi grey synthetic evaluation on pavement condition of asphalt pavement", Bulletin Of Science And Technology, vol. 25, no. 1, pp. 98-102, 2009.
[13] G. Sheng-fei, L. Jian-ming, and Z. Hong-duo, "Matter element model for PPM Treatment selection of highway asphalt pavement", Journal of Kunming University of Science and Technology (Science and Technology), vol. 32, no. 5, pp. 73-77,81, 2007.

[14] H. Qun-fang, L. Wen, and L. Qing-fu, "Synthetical gray evaluation of asphalt pavement performance", Journal of Highway and Transportation Research and Development, vol. 23, no. 1, pp. 12-15, 2006.

[15] L. Qiang, and W. Jing-gang, "Testing and evaluation of asphalt pavement structural performance", Journal of Highway and Transportation Research and Development, vol. 23, no. 2, pp. 23-26, 2006.

(C) Zhi-bin et al.; Licensee Bentham Open.

This is an open access article licensed under the terms of the (https://creativecommons.org/licenses/by/4.0/legalcode), which permits unrestricted, noncommercial use, distribution and reproduction in any medium, provided the work is properly cited. 